Case report

\title{
Plasty of Achilles region with propeller tendon- fasciocutaneous peroneal artery perforator flap after radical excision of a neglected squamous cell carcinoma
}

\author{
Viorica Mihaluța*,1, Alina Stoian ${ }^{1}$, Rodica Iordăchescu², Elena Pavlovschi ${ }^{2}$, Adrian \\ Cociug $^{3}$, Eleonora Borș ${ }^{1}$, Snejana Smolenschi ${ }^{4}$, Grigore Verega ${ }^{1}$ \\ 1"Nicolae Testemițanu" State University of Medicine and Pharmacy, Chişinău, Republic of Moldova; \\ ${ }^{2}$ IMSP Institute of Emergency Medicine, Plastic Surgery and Reconstructive Microsurgery \\ Department, Chişinău, Republic of Moldova; ${ }^{3}$ The Human Research Tissue Bank, Chişinău, Republic \\ of Moldova; ${ }^{4}$ Department of Plastic Surgery, Regional Institute of Oncology, Iaşi, Romania
}

\begin{abstract}
Squamous cell carcinoma (SCC) is a slow-growing, aggressive skin cancer with tendency for recurrence and metastasis to the lymph nodes and adjacent vital structures. Treatment for SCC is wide excision and reconstruction. Complex defects with tendon or bone exposure require reconstruction with local, pedicled or free flaps, either cutaneous, fascial or tendon-fasciocutaneous, bone or muscle flaps. The aim of the case report is to present the post-oncologic reconstruction of the complex defect of Achilles region using a composite tendon-fasciocutaneos peroneal propeller pedicled perforator flap with anatomic and functional restoration. Case report: a 57-year-old man with diabetes type II for 10 years, presented with a 20-year history of static non-healing ulcer at left Achilles tendon level after a car accident. The biopsy revealed squamous cell carcinoma on the background of chronic inflammation. Wide resection included Achilles tendon and calcaneus bone, with resulting tissue defect of $12 \times 16 \mathrm{~cm}$. Perforator peroneal artery flap with tendon of $11 \mathrm{~cm}$ length rotated propeller in 180 degree to the distal region provided defect coverage. Skin graft resurfaced the donor site. Postoperative recovery was uneventful, the tendon-fasciocutaneous flap survived entirely with skin graft fully integrated. Conclusions: The tendon-fasciocutaneous peroneal artery perforator flap in pedicled propeller fashion provides good function of the limb with minimum recovery time for oncologic patients. Concomitantly, the cosmetic aspect of the reconstruction makes our choice a valuable choice to resurface complex defects of the Achilles region.
\end{abstract}

Keywords: squamous cell carcinoma; Achilles region; tendon-fasciocutaneous peroneal artery perforator; reconstructive surgery; propeller flap

\section{Introduction}

Squamous cell carcinoma (SCC) is one of the most common forms of the skin cancer that originates from the epithelium [1, 2]. Cutaneous SCC develops more frequently in chronically injured or long-standing skin diseases, for example in burn patients or in osteomyelitis. In most instances, this type of SCC is more aggressive locally, invading the

Received: July 2018; Accepted after review: September 2018; Published: September 2018.

${ }^{*}$ Corresponding author: Viorica Mihaluța, "Nicolae Testemițanu" State University of Medicine and Pharmacy, 165, Ștefan cel Mare și Sfânt Bd. MD-2004, Chişinău, Republic of Moldova

Email: mihalutaviorica@yahoo.com surrounding anatomical structures earlier, with higher chances for recurrence and increased metastatic potential [3]. The clinical presentations of the chronic ulcers and squamous cell carcinomas can vary, but at the same time they can coexist $[4,5]$.

The aim is to report a clinical case of a post-oncological reconstruction after a 20years non-healing ulceration of a large defect at Achilles level. The reconstruction was performed with tendon-fasciocutaneous peroneal artery perforator flap, in a propeller manner translation, with good function of the limb with minimum recovery time. 


\section{Case report}

A 57 year-old man with diabetes type II for 10 years was admitted to our department with an ulcerated tumor of $5 \times 10 \mathrm{~cm}$ at the Achilles level with functional impotence, developed on a non-healing ulcer at the Achilles level after 20 year-old car accident (Figure 1).

Preoperatively, the cutaneous arteries were evaluated using a hand-held Doppler (Figure 1) and the reconstruction plan was designed to include posterior calf skin and advance it in propeller fashion to cover the post-excisional defect (Figure 2). The patient agreed to the proposed treatment.

The tumor was removed along with minimum $1 \mathrm{~cm}$ healthy tissue circumferentially and $1 \mathrm{~cm}$ deep margin even in the bone, resulting in a $3 \mathrm{~cm}$ deep bony defect with absence of overlying Achilles tendon. Multiple specimens were harvested for frozen section evaluation.
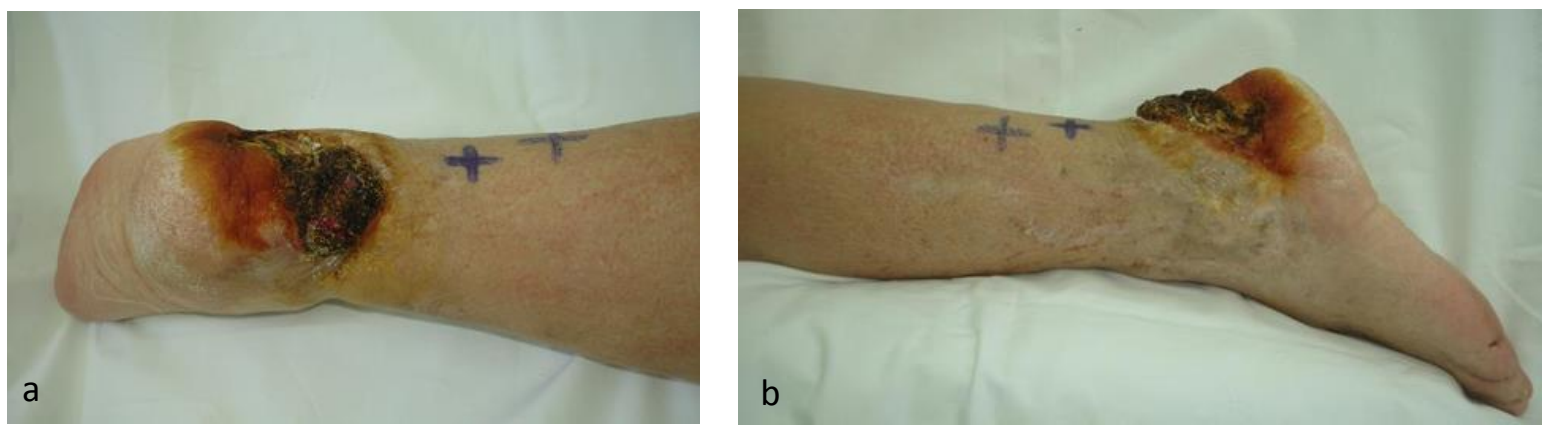

Fig. 1. Preoperative images: a) posterior-anterior view of the Achilles level with markings of the skin projection of peroneal artery perforators on Doppler examination; b) lateral view.

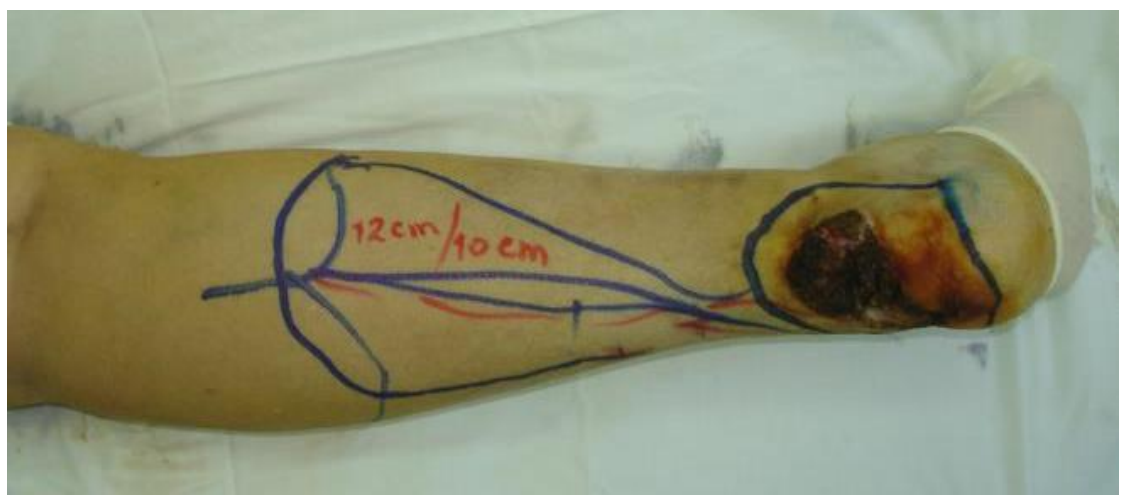

Fig. 2. Drawing the incision patterns. The size of the flap is $12 \times 10 \mathrm{~cm}$
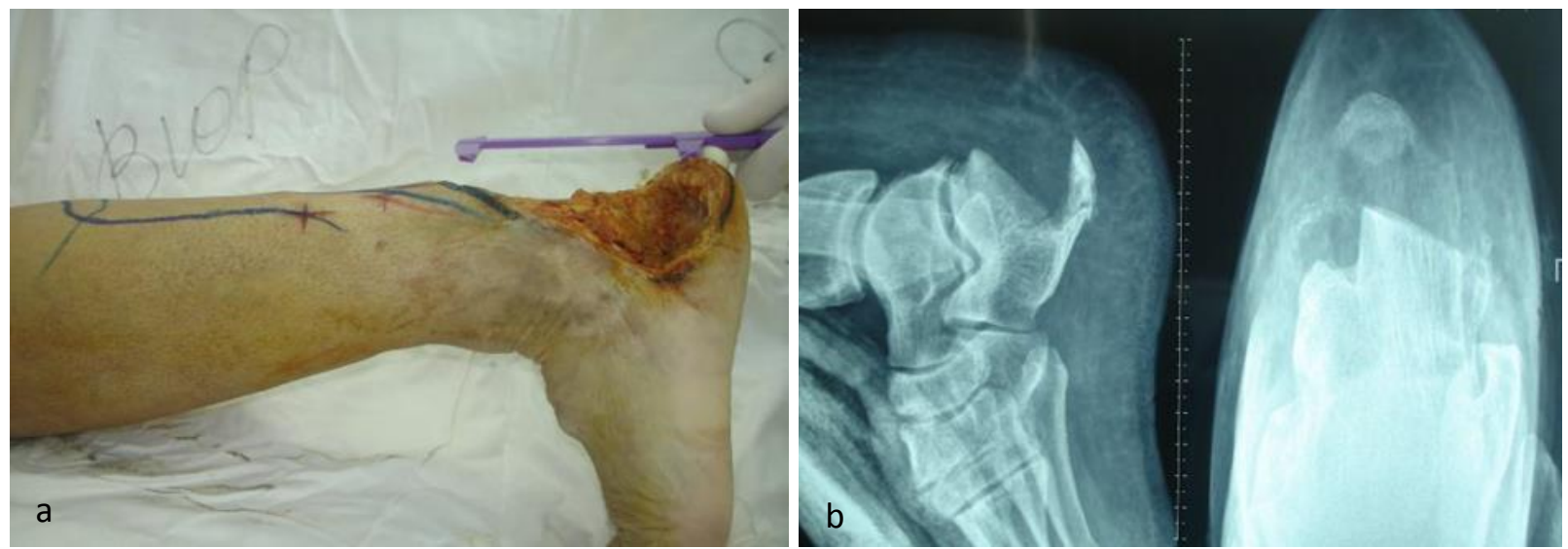

Fig. 3. The image of the radical resection of the: a) distal Achilles tendon and soft tissue defect; b) bone-tissue defect of $3 \mathrm{~cm}$. 
Calf skin was incised according to the preoperative plan (Figure 3). Fascia was included together with distal medial half of the Achilles tendon for reconstruction of the $11 \mathrm{~cm}$ tendon defect (Figure 4). The flap was harvested on peroneal perforator vessels marked on the skin (Figure 1). The composite tendonfasciocutaneous flap was rotated $180^{\circ}$ to the distal region and inset.

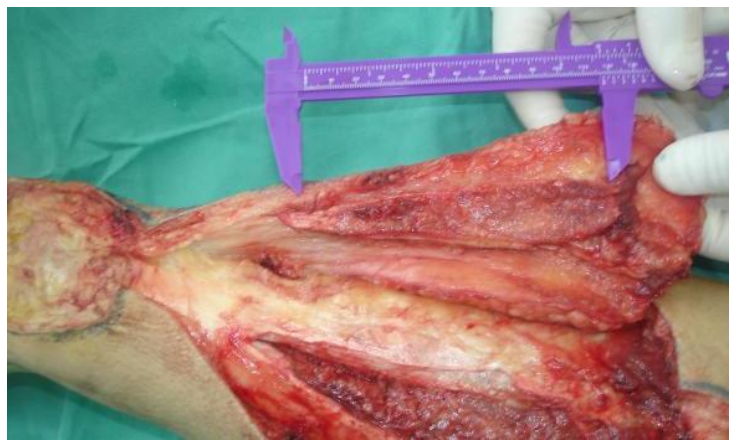

Fig. 4. The composite fasciocutaneous and tendon flap harvested, pedicled on the perforator vessels. Tendon graft is showed in the ruler.
The tendon included in the flap reconstructed the gap of the distal Achilles tendon overlying the calcaneus, the skin island covered the defect and donor site defect was skin grafted.

The patient underwent large excision of the tumor and the complex defect including soft tissue, tendon and bone was reconstructed using flap from calf area. The frozen sections identified no cancer in the resection margins after the excision. The final pathological examination confirmed squamous cell carcinoma on the background of chronic inflammation; the bone tissue presented necrosis (Figure 5). The excision margins were free of cancer cells.

The postoperative period was uneventful, with survival of the entire flap. The ankle joint function was restored to dorsal flexion $-10^{\circ}$, plantar flexion $-25^{\circ}$, the patients was ambulating normally, without pain (Figure 6).

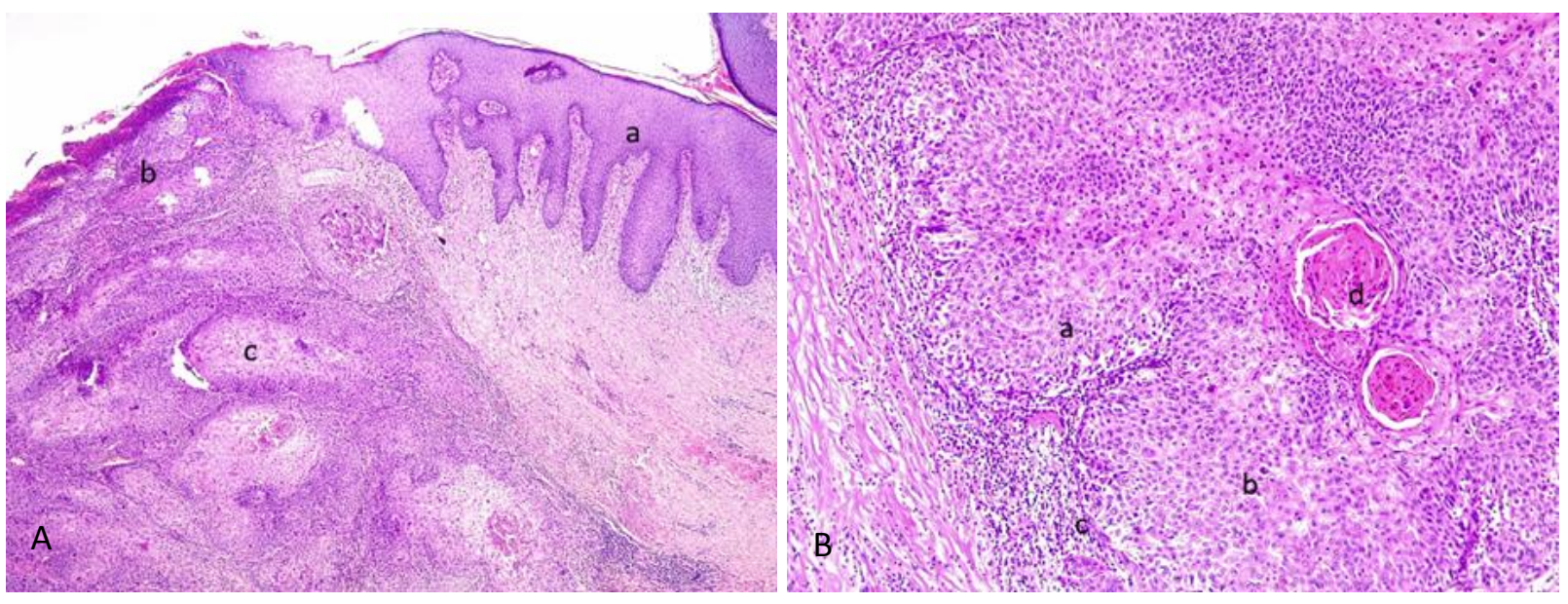

Fig. 5. A) Squamous cell carcinoma: Thickening of the epidermis with papillomatosis (a), epidermal detachment and ulceration (b), carcinomatous infiltration surrounded by inflammatory infiltrate (c) and intradermal keratin pearls (HE, x40); B) Neoplastic islands with cellular atypia (a), cells with increased mitotic activity (b), inflammatory infiltrate (c) and keratin pearl formation (d) (HE, x100). 

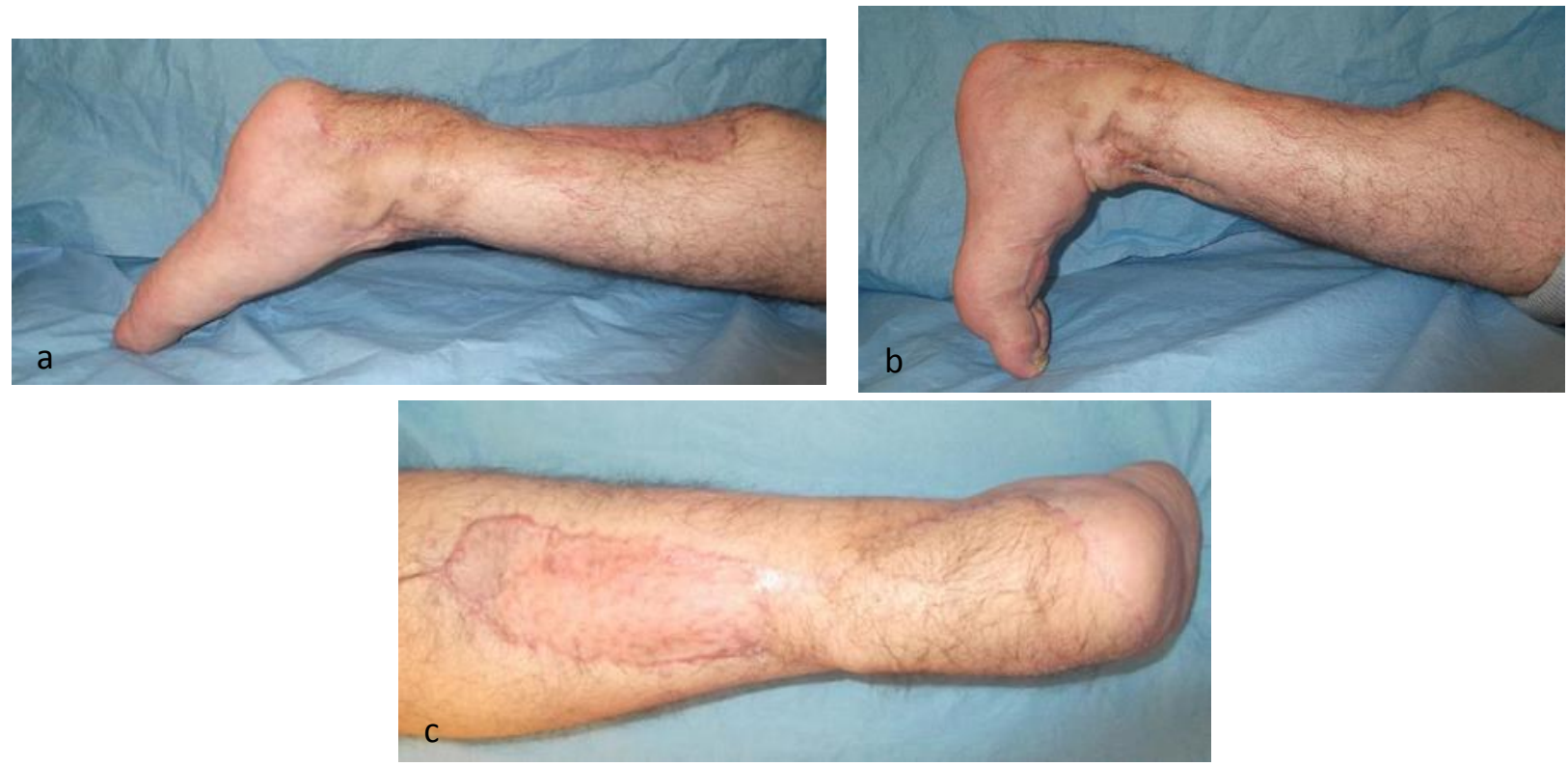

Fig. 6. a) Plantar flexion $-25^{\circ}$; b) Dorsal flexion $-10^{\circ}$; c) Achilles region aspect one month postoperatively with acceptable contouring of the flap inset in the defect and of the skin graft intake.

\section{Discussion}

Our case report is about a diabetic patient with squamous cell carcinoma developed on a chronic inflammatory wound persisting for 20 years following a car accident. Malignant transformation from chronic wounds is notorious, with up to $25 \%$ of cancers being linked to inflammation or infection, either viral or bacterial. Moreover, many authors report that epidermal cells are highly susceptible to malignant transformation and cancer progression to metastatic disease status [6-9].

Following SCC treatment protocols, the patient underwent wide excision with minimum $1 \mathrm{~cm}$ macroscopic circumferential and deep margins which resulted in a complex defect involving bone, tendon and soft tissue.

Reconstruction of complex defects of Achilles region is challenging and aims to achieve soft tissue stable coverage, restoration of limbs' form and function and pleasing cosmetic effect in a minimum recovery timeframe. Of course, ambulation is the most important function therefore it should not be impeded by a bulky flap [10].

The main reconstruction options of Achilles region are the free tissue transfer and the loco-regional flaps, with the latter being increasingly used with the recent progress of perforator flaps. Traditional free muscle flaps are latissimus dorsi and gracillis while fasciocutaneous flaps, while the most frequently used are the lateral arm and the anterolateral thigh flap. The choice depends mostly of patients' needs in terms of defect size, composition, comorbidities but equally on surgeons' preference and expertise [11, 12].

In our patient, because of the long history of diabetes and chronic wound, we looked for a solution to avoid diabetic angiopathy complications and the probable complications linked to microsurgery, to spare the main vessels of the leg and to avoid long operating hours. A loco-regional fascial flap could be a simple and safe alternative for a free flap, provided it should be thin, pliable and have a sufficient amount to cover the defect while concomitantly reconstructing the tendon defect [13].

Perforator flaps rose as an alternative to free tissue transfer in patients in whom the complications toll is high: comorbidities including peripheral vascular disease, thrombosis and ischemia, loss of major vessels of the limb to microsurgery or extensive trauma or multiple surgeries, partial or total flap loss. Moreover, they are thin when compared to muscle or musculo-cutaneous flaps; they are based on short, small-caliber 
side-branches of the main vessel and not on main vessel itself, therefore produce less donor-site morbidity. The distal vessels perforator flaps are the solutions for defects located in distal two-thirds of the calf including Achilles area and the heel [14-16].

Over the free flaps, the peroneal artery perforator flap has notable advantages making it a suitable option for distal leg defects: reliable, versatile design, composition, size and mobility of the flap, shorter operating time, less bleeding and thrombosis, calf muscle and peroneal vessel sparing while providing adequate blood perfusion to the flap [17, 18].

\section{References}

1. Nagy V. Principii de cancerologie generală. Curs pentru studenţi. Cluj-Napoca: Editura Medicală Universitară Iuliu Haţeganu, 2007; 37-47.

2. Stephens FO, Aigner KR. Basics of oncology. Springer, Heidelberg, London, New York, 2009; 49-64.

3. Kirsner RS, Spencer J, Falanga V, Garland LE, Kerdel FA. Squamous cell carcinoma arising in osteomyelitis and chronic wounds. Treatment with Mohs micrographic surgery vs amputation. Dermatol Surg 1996; 22(12):1015-1018.

4. Cassarino DS, Derienzo DP, Barr RJ. Cutaneous squamous cell carcinoma: a comprehensive clinicopathologic classification. Part one. J Cutan Pathol 2006; 33(3):191-206.

5. Enoch S, Miller DR, Price PE, Harding KG. Early diagnosis is vital in the management of squamous cell carcinomas associated with chronic non healing ulcers: a case series and review of the literature. Int Wound $J$ 2004; 1(3):165-175.

6. Tang L, Wang K. Chronic inflammation in skin malignancies. J Mol Signal 2016; 11:2.

7. Hussain SP, Harris CC. Inflammation and cancer: an ancient link with novel potential. Int J Cancer 2007; 121:2373-2380.

8. Mantovani A, Allavena $P$, Sica A, Balkwill F. Cancer-related inflammation. Nature 2008; 454:436-444.

9. Aggarwal BB, Gehlot $P$. Inflammation and cancer: how friendly is the relationship for cancer patients? Curr Opin Pharmacol 2009; 9:351-369.

10. Chalmers R, Tare M, Niranjan N. Vascularized Achilles tendon graft reconstruction - by the

\section{Conclusions}

The composite tendon-fasciocutaneous peroneal perforator flap can be used distally in pedicled propeller fashion to reconstruct defects of the calf and calcaneus in oncologic cases. It provides good functional reconstruction and aesthetically pleasing results with minimum recovery time. We do recommend the use of the flap in the armamentarium of reconstruction ladder for distal calf area.

\section{Competing interests}

The authors have no conflict of interests to declare.

Patient consent

Written informed consent was obtained from the patient's legal guardians for publication of this case.

tendon for the tendon. $J$ Plast Reconstr Aesthet Surg 2010; 63:273-276.

11. Sabapathy SR, Venkataramani $H$, Latheef $L$, Bhardwaj P. Reconstruction of segmental defects of Achilles tendon: Is it a must in infected complex defects? Indian J Plast Surg 2013; 46:121-123.

12. Wei FC, Chen HC, Chuang CC, Noordhoff MS. Reconstruction of Achilles tendon and calcaneus defects with skin-aponeurosis-bone composite free tissue from the groin region. Plast Reconstr Surg 1988; 81:579-589.

13. Upton J, Tracy MB, Steven LS, et al. Fascial flap coverage of Achilles tendon defects. Plast Reconstr Surg 1995; 95:1056-1061.

14. Lin CT, Chen CY, Chang SC. Bipedicled flap reconstruction of soft tissue defect with Achilles tendon exposure. J Med Sci 2014; 34:129-132.

15. Michlits W, Gruber S, Windhofer C, Macheiner P, Walsh M, Papp C. Reconstruction of soft tissue defects overlying the Achilles tendon using the super extended abductor hallucis muscle flap. J Trauma 2008; 65:1459-1462.

16. Baj A, Ali Youssef $D$, Monteverdi $R$, et al. Recontruction of partial maxillary defects with the double-barrel fibula free flap. Acta Otorhinolaryngol Ital 2010; 30:299-302.

17. Vaienti L, Marchesi A, Palitta G, Gazzola R, Parodi PC, Leone F. Limb trauma: the use of an advanced wound care device in the treatment of full-thickness wounds. Strateg Trauma Limb Reconstr 2013; 8:111-115.

18. Cheng L, Yang $X$, Chen $T$, Li Z. Peroneal artery perforator flap for the treatment of chronic lower extremity wounds. J Orthop Surg Res 2017; $12: 170$ 